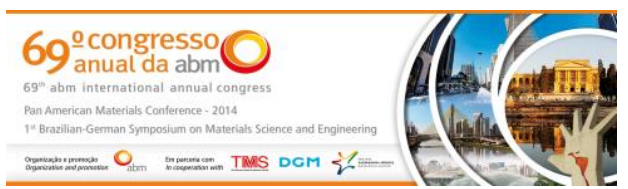

Tema: Nanotecnologia

\title{
CARATERIZAÇÃO MICROESTRUTURAL E DA TEXTURA CRISTALOGRÁFICA DA LIGA AI AA1070 DEFORMADO A FRIO VIA ECAP A PARTIR DA ROTA C*
}

\section{Resumo}

Gabriel Gonçalves Pessoa de Castro ${ }^{1}$ Rachel dos Santos Mendes ${ }^{1}$ Manuela Fontana ${ }^{2}$ Ana Carolina Ribeiro Duarte ${ }^{1}$ Jefferson Fabrício Cardoso Lins $^{3}$

O trabalho teve por objetivo produzir uma liga Al AA1070 com microestrutura ultrafina via prensagem por canais equiangulares. O processo empregou a rota $C$ com 2 passes consecutivos a frio e acumulou uma deformação $\varepsilon_{2}=2,38$. O material de partida é proveniente de um processo de laminação a quente na qual a microestrutura resultante era composta de grãos grosseiros com tamanho de grão de 14,5 $\pm 19,1 \mu \mathrm{m}$ e dureza de $31,1 \pm$ 1,9 Vickers. Foi realizada a caracterização microestrutural com o auxílio das técnicas de microscopia eletrônica de varredura (MEV) e de difração de elétrons retroespalhados. $O$ comportamento mecânico das amostras foi avaliado por ensaios de dureza Vickers, a fim de se determinar a energia armazenada durante os passes. Os resultados obtidos mostraram que o material de partida apresentou estrutura parcialmente recristalizada, decorrente do processo termomecânico de laminação. A deformação cisalhante severa que ocorre na interseção entre os canais durante o processamento via ECAP permitiu um intenso refinamento de grãos. $O$ tamanho de grão após o $2^{\circ}$ passe foi da ordem de 1,6 $\pm 1,7 \mu \mathrm{m}$. A dureza após o $1^{\circ}$ passe quase dobrou em relação ao material de partida, mas praticamente se manteve no passe seguinte, sugerindo uma possível estabilidade. Pode-se concluir que ocorreu um considerável refinamento do tamanho de grão em função da elevada fragmentação e rotação da subestrutura após os passes de deformação.

Palavras-chave: MEV; ECAP; Al AA 1070; Deformação plástica severa; Caracterização microestrutural e textura cristalográfica.

\section{MICROSTRUCTURAL CHARACTERIZATION AND OF THE CRYSTALLOGRAPHIC TEXTURE OF THE AL AA1070 ALLOY DEFORMED BY THE COLD ECAP PROCESS FROM ROUTE C Abstract}

The study aimed to produce an alloy with ultrafine Al AA1070 via equiangular channels by pressing microstructure. The process employed to route $C$ with 2 consecutive cold passes and accumulated deformation $\varepsilon 2=2.38$. The starting material is from a hot rolling process in which the resulting microstructure consisted of coarse grains with grain size of $14.5 \pm 19.1$ micrometers me hardness of $31.1 \pm 1.9$ Vickers. Microstructural characterization was performed with the aid of electron microscopy (SEM) and electron backscatter diffraction. The mechanical behavior of the samples was measured by Vickers hardness tests in order to determine the energy stored during the passes. The results showed that the starting material had partially recrystallized structure, resulting from the thermomechanical rolling process. The severe shear deformation that occurs at the intersection between channels during processing via ECAP allowed an intensive grain refinement. The grain size after the 2 nd pass was approximately $1.6 \pm 1.7$ micrometers. The hardness after the 1st pass almost doubled compared to the starting material, but virtually remained the next pass, suggesting a possible stability. It can be concluded that there was a considerable refinement of grain size due to the high fragmentation and rotating the substructure passes after deformation.

Keywords: MEV; ECAP; Al AA 1070; Severe plastic deformation; Microstructural characterization and crystallographic texture.

1 Aluno-Cursando Mestrado em Engenharia Metalúrgica, Universidade Federal Fluminense (UFF), Volta Redonda, RJ, Brasil.

2 Aluno-Cursando Doutorado em Engenharia Metalúrgica, UFF, Volta Redonda, RJ, Brasil.

3 Professor Orientador, Doutor em Engenharia Metalúrgica, Universidade Federal Fluminense, Volta Redonda, RJ, Brasil.

\footnotetext{
* Contribuição técnica ao 69 Congresso Anual da ABM - Internacional e ao 14ํㅡㄹ ENEMET - Encontro Nacional de Estudantes de Engenharia Metalúrgica, de Materiais e de Minas, 21 a 25 de julho de 2014, São Paulo, SP, Brasil.
} 


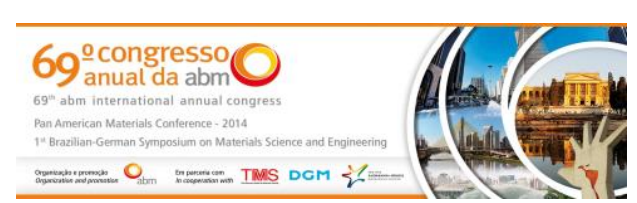

\section{INTRODUÇÃO}

A deformação plástica severa (DPS), através de um intenso processo de refinamento microestrutural de materiais como cobre, titânio, ligas de alumínio e aços ligados, vem se mostrando uma técnica eficaz para obtenção de materiais com propriedades superiores $[1,2]$.

O surgimento de microestruturas ultrafinas e a manipulação da estrutura dos materiais numa escala nanométrica utilizando técnicas de deformação não convencionais, é objetivo de estudo da área da ciência intitulada como "nanotecnologia incremental" [3].

Dentre as técnicas não convencionais de DPS destaca-se a prensagem em canais equiangulares (ECAP) inventada por Segal em 1972 [4], que consiste na passagem de um tarugo por canais equiangulares com secção transversal constante, onde é aplicado sobre o material uma deformação de cisalhamento simples que promove o surgimento de microestruturas especiais. Este processo pode ser conduzido em quatro rotas de processamento, nomeadas de rotas $\mathrm{A}, \mathrm{BA}, \mathrm{BC}$ e $\mathrm{C}$, que favorecem a ativação de diferentes sistemas de deslizamento produzindo diferenças na microestrutura do material processado.

A distinção entre as rotas do ECAP esta entre as rotações do material após cada passe de deformação. Na rota $A$ o material processado não sofre rotação entre os passes de deformação. Na rota BA o material sofre rotações alternadas de $90^{\circ}$, em relação à direção de prensagem, nos sentidos horário e anti-horário. Na rota $\mathrm{BC} 0$ material sofre após cada passe uma rotação de $90^{\circ}$ no sentido horário. Por fim, na rota $\mathrm{C}$ o material sofre uma rotação de $180^{\circ}$ entre cada passe.

Estudar materiais pouco investigados, como a liga de alumínio Al AA1070, pode esclarecer algumas dúvidas sobre os efeitos no refinamento da microestrutura pelas diferentes rotas de processamento. Tolaminyad and Dehghani [5] realizaram a caracterização microestrutural e avaliaram algumas propriedades mecânicas dessa liga via ECAP através da rota Bc. Foi verificado dois tipos de comportamento, o primeiro ao quarto passe onde a microestrutura evoluiu de subgrãos alongados para uma matriz de grãos ultrafinos e equiaxiais e que a fração de contornos de alto ângulo e a diferença cristalográfica cresceram rapidamente até o quarto passe. Do quarto ao oitavo passe a velocidade de crescimento se reduziu e não foi notada nenhuma alteração significativa no tamanho dos grãos.

Neste contexto, a investigação da liga Al AA1070 consiste na análise sistemática de seu comportamento durante a deformação via ECAP ao longo de 2 passes de deformação utilizando a rota $\mathrm{C}$ do ECAP, através da caracterização microestrutural, da textura cristalográfica e ensaios mecânicos. A caracterização microestrutural do material após sofrer DPS aplicada por ECAP via rota C foi avaliada através de microscopia eletrônica de varredura (MEV). A microtextura e a macrotextura foram obtidas através da técnica de difração de elétrons retroespalhados (EBSD). 0 comportamento mecânico das amostras foram avaliados por ensaios de dureza Vickers e de compressão uniaxial quase-estática, a fim de quantificar a energia armazenada e comparar a evolução das propriedades mecânicas e do refino dos grãos do material após cada passe de deformação.

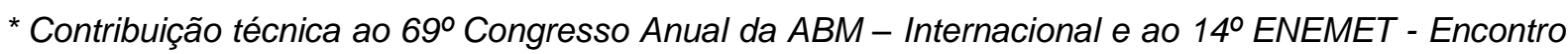
Nacional de Estudantes de Engenharia Metalúrgica, de Materiais e de Minas, 21 a 25 de julho de 2014, São Paulo, SP, Brasil.
} 


\section{MATERIAIS E MÉTODOS}

\subsection{Material}

O corpos de prova utilizados no proposto trabalho são provenientes de uma liga de alumínio AA 1070, fornecida pela empresa Novelis do Brasil Ltda., cuja composição química é apresentado na tabela 1. A chapa foi lingotada para a confecção de uma placa de $610 \mathrm{~mm}$ de espessura e em seguida, desbastada via laminação a quente até a espessura de $32 \mathrm{~mm}$ cuja temperatura de saída foi estimada como sendo acima de $380^{\circ} \mathrm{C}$.

Tabela 1. Composição química da liga de alumínio AA1070 (Teores expressos em \% de peso)

\begin{tabular}{|c|c|c|c|c|c|c|c|c|}
\hline $\mathbf{M n}$ & $\mathbf{M g}$ & $\mathbf{S i}$ & $\mathbf{P b}$ & $\mathbf{F e}$ & $\mathbf{T i}$ & $\mathbf{C u}$ & $\mathbf{G a}$ & $\mathbf{A l}$ \\
\hline- & - & 0,07 & 0,002 & 0,18 & 0,02 & - & 0,001 & 99,72 \\
\hline
\end{tabular}

\subsection{Método}

\subsubsection{Processo ECAP}

No processamento ECAP tarugos de alumínio com formato de paralelepípedos retangulares (tarugos) com dimensões de $70 \times 10 \times 10 \mathrm{~mm}$, serão forçados a escoar por dois canais de secção transversal igual e constante no interior de uma matriz totalmente rígida, sofrendo deformações de natureza de cisalhamento simples conforme passam pela zona de interseção entre os dois canais. Os canais foram lubrificados com graxa de alto desempenho de sulfonato de cálcio. A rota utilizada no processamento é a rota $\mathrm{C}$, que consiste em girar o corpo $180^{\circ}$ após cada deformação, foram realizados 2 passes.

O processamento ECAP foi realizado no Laboratório de Ensaios Mecânicos da EEIMVR/UFF, utilizando uma máquina de Tração e Compressão universal EMIC DL60 com capacidade máxima de carga de 600 KN, uma matriz ECAP bipartida de aço ferramenta $\mathrm{H} 13$ com dois canais idênticos com dimensões de $10 \times 10 \mathrm{~mm}$ formando um ângulo de $90^{\circ}$ e um punção de aço ferramenta $\mathrm{H} 13$;

O raio de adoçamento dos canais é de $5 \mathrm{~mm}\left(\Psi \approx 37^{\circ}\right)$ a fim de facilitar o fluxo do tarugo entre os canais e reduzir a carga de prensagem. O processo de deformação ocorreu a uma velocidade de $5 \mathrm{~mm} / \mathrm{min}$ e acumulou uma deformação verdadeira de $\varepsilon 2=2,38$.

\subsubsection{Preparação metalográfica}

As amostras deformadas via processamento ECAP após cada ciclo e uma amostra sem deformação, foram cortadas, com dimensões aproximadas de $10 \times 10 \times 5 \mathrm{~mm}^{3}$ para a análise microestrutural. Primeiramente as amostras forma lixadas e em seguida submetidas ao polimento eletrolítico. O polimento foi realizado através de duas células eletrolíticas no equipamento padrão, empregando-se uma solução composta por 59 partes de $\mathrm{CH}_{4} \mathrm{O}$ (metanol), 35 partes de $\mathrm{C}_{2} \mathrm{H}_{4}(\mathrm{OH})_{2}$ (etileno-glicol) e 6 partes de $\mathrm{HClO}_{4}$ (ácido perclórico) (em volume). A diferença de potencial (ddp) aplicada foi de $8 \mathrm{~V}$ por 90 segundos a temperatura ambiente.

\subsubsection{Microscopia eletrônica de varredura - MEV}

A microestrutura das amostras foi observada e avaliada com auxílio de um microscópio eletrônico de varredura EVO MA10 da Zeiss com filamento $\mathrm{LaB}_{6}$ que se

\footnotetext{
* Contribuição técnica ao $69^{\circ}$ Congresso Anual da ABM - Internacional e ao 14ํㅡㄹ ENEMET - Encontro Nacional de Estudantes de Engenharia Metalúrgica, de Materiais e de Minas, 21 a 25 de julho de 2014, São Paulo, SP, Brasil.
} 
encontra instalado no Laboratório Multiusuário de Microscopia Eletrônica (LMME) da EEIMVR/UFF.

\subsubsection{Difração de elétrons retroespalhados - EBSD}

As medidas de microtextura e mesotextura foram realizadas com o auxílio da técnica de Difração de Elétrons Retroespalhados (EBSD) e o sistema do modelo Pegasus MX4i da marca EDAX, com câmera de alta velocidade modelo HIRAKI, integrado ao MEV. Uma área com dimensões aproximadas de $60 \times 100 \mu^{2}$ de cada amostra foi mapeada, de acordo com a morfologia dos elementos da subestrutura presentes, com um passo de varredura (step size) variando de 0,1 a 0,3 $\mu \mathrm{m}$.

As informações geradas por essas varreduras serão integradas e processadas pelo software OIM 5.3 da EDAX.

\subsubsection{Dureza de Vickers}

A fim de se determinar a energia armazenada, de maneira indireta, na seção transversal das amostras, foi realizado ensaio mecânico de dureza de Vickers, através de um microdurômetro da marca Shimadzu modelo HVM-2T instalado no LMME na EEIMVR/UFF, as impressões Vickers serão realizadas com carga de 0,1 kgf durante $30 \mathrm{~s}$.

\section{RESULTADOS E DISCUSSÃO}

Os resultados são apresentados por meio de micrografias obtidas com auxílio de microscopia eletrônica de varredura. Também são apresentadas estimativas de energia armazenada realizadas por meio de medidas de dureza Vickers.

As curvas de Tensão X Deformação dos passes 1 e 2 do ECAP via rota $C$ são apresentados na Figura 1, onde é observado um aumento de tensão de deformação no segundo passe, resultante do crescimento de sua resistência mecânica, proveniente do aumento energia interna do material após o primeiro passe de deformação.

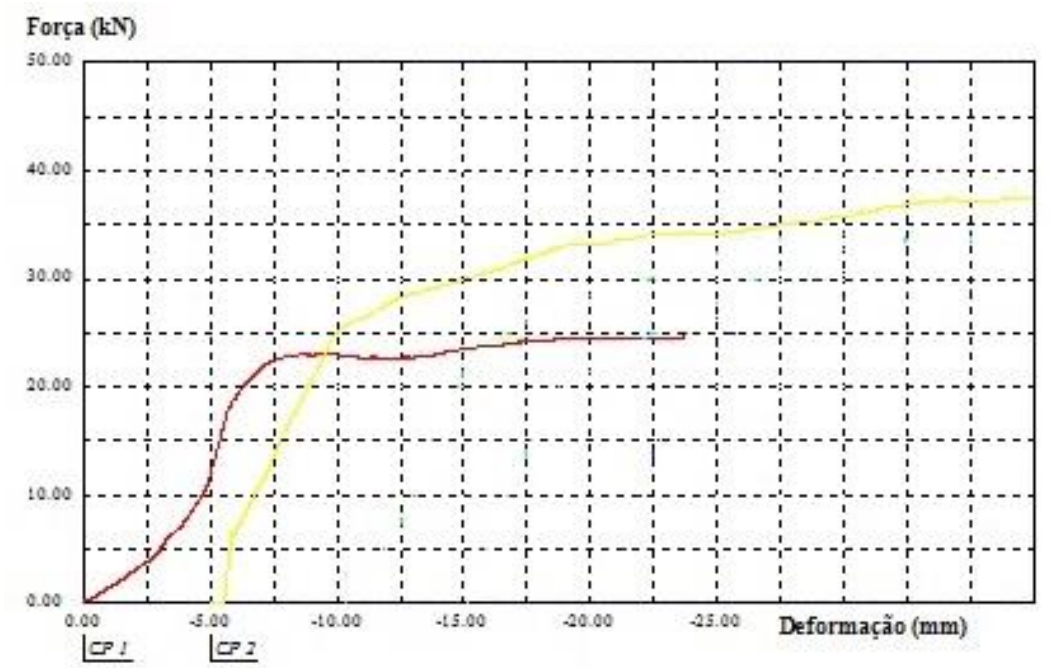

Figura 1. Gráfico Tensão X Deformação do primeiro e segundo passe ECAP - Rota C.

\footnotetext{
* Contribuição técnica ao $69^{\circ}$ Congresso Anual da ABM - Internacional e ao 14ํㅡㄹ ENEMET - Encontro Nacional de Estudantes de Engenharia Metalúrgica, de Materiais e de Minas, 21 a 25 de julho de 2014, São Paulo, SP, Brasil.
} 


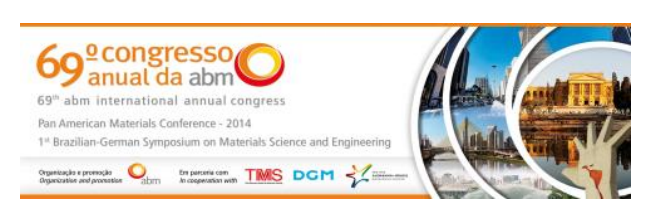

\subsection{Material de Partida}

A liga de alumínio AA1070 laminada a quente utilizada no presente trabalho, foi caracterizada por Rezende e colaboradores [6], foi observado grãos com morfologia e tamanho irregular, com uma elevada fração de contornos com caráter de baixo ângulo, cerca de 55\% em sua secção transversal. A Figura 2 apresenta os mapas de orientação, onde é possível observas o crescimento anormal de grãos, ou recristalização secundária. As medidas de tamanho de grão realizadas por EBSD na seção transversal obtiveram como medida o valor de 14,5 $\pm 19,1 \mu \mathrm{m}$. Observa-se um desvio elevado, que se deve como podemos observar na Figura 1, a presença de grãos muito grosseiros em sua microestrutura. Os grãos com crescimento exagerado exibem formato equiaxial e chegam a medir $132 \mu \mathrm{m}$, essa heterogeneidade também foi observada por Alvi e colaboradores [7] ao estudar e caracterizar uma liga de alumínio comercialmente pura AA1050, onde observaram também a existência de grãos muito maiores que a média. $O$ material apresentou uma dureza de $31,1 \pm 1,9$ Vickers.

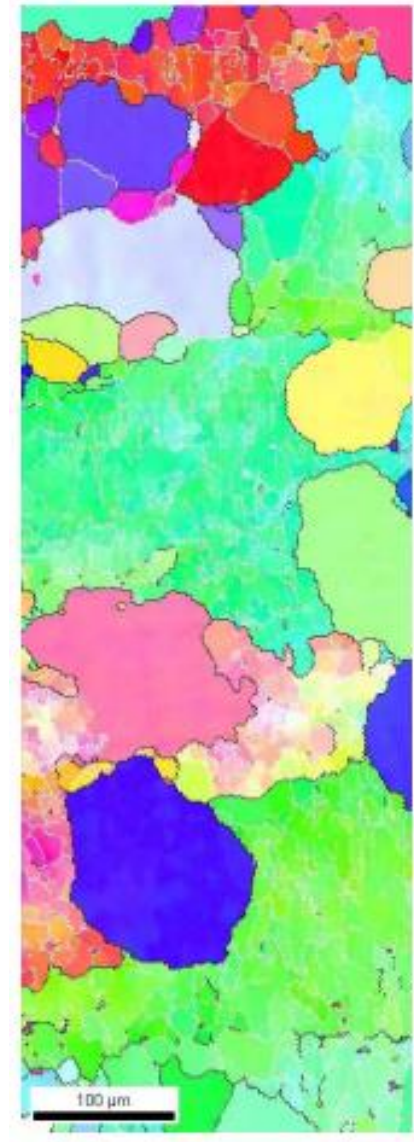

a)

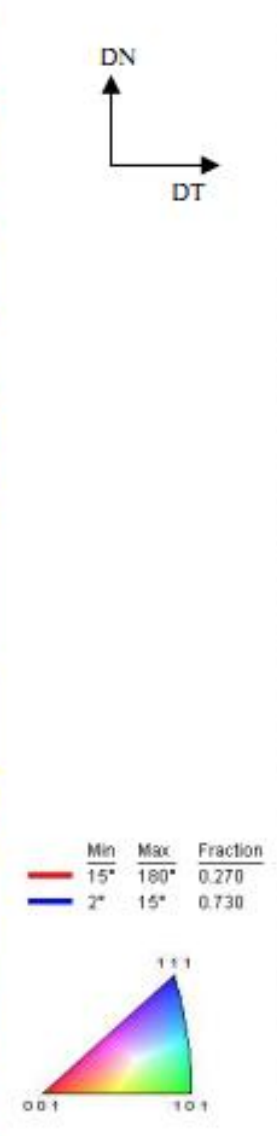

b)

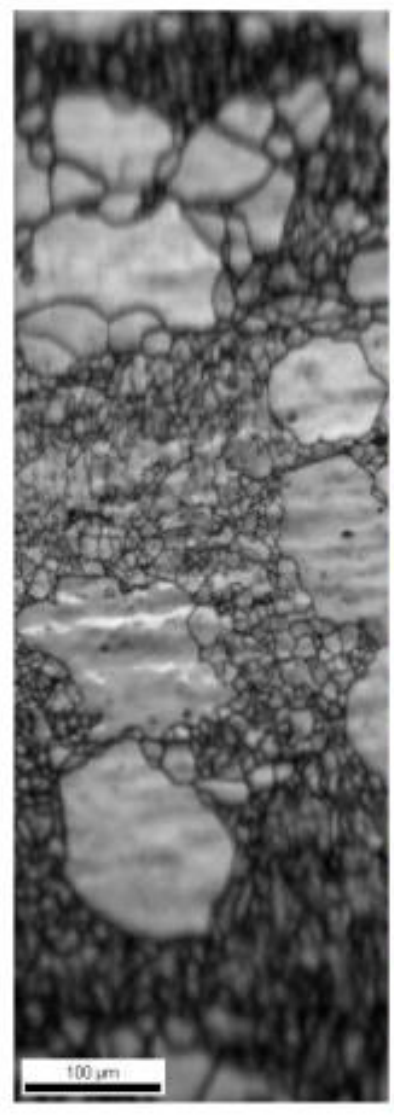

b)

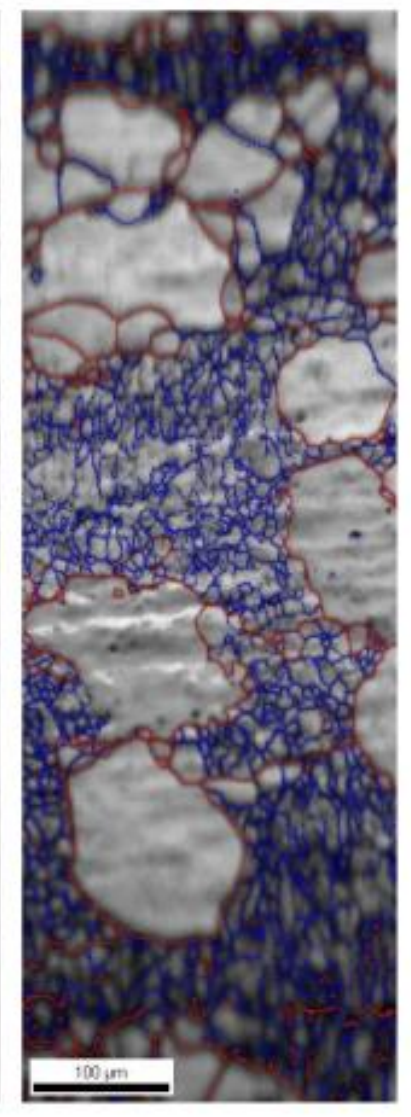

c)

Figura 2. Imagens da seção transversal do material de partida: a) Mapa de orientação; b) Mapa de qualidade; c) Mapa de qualidade com contornos delineados. (MEV - EBSD, 20 kV) [9].

\subsection{Primeiro Passe de Deformação - Rota C}

Após o processamento via ECAP a microestrutura da liga AA1070, previamente laminada a quente, mudou consideravelmente, sua estrutura foi altamente refinada,

\footnotetext{
* Contribuição técnica ao $69^{\circ}$ Congresso Anual da ABM - Internacional e ao 14ํㅡㄹ ENEMET - Encontro Nacional de Estudantes de Engenharia Metalúrgica, de Materiais e de Minas, 21 a 25 de julho de 2014, São Paulo, SP, Brasil.
} 


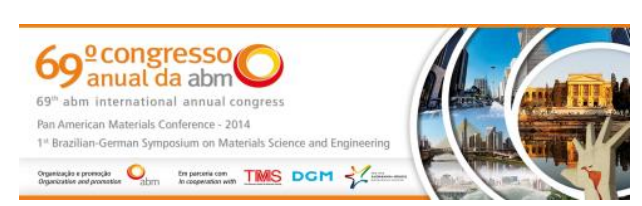

e foi um crescimento na fração de contornos de baixo ângulo juntamente com um grande aumento na energia interna armazenada, identificado pelo aumento de sua dureza.

A fração de contornos de baixo ângulo é apresentada na Figura 3 e corresponde a $77 \%$, e $23 \%$ de contornos de alto ângulo, um aumento significativo quando comparado ao material de partida, cerca de 55\%. Kawasaki e seus colaboradores [8], estudando alumínio de alta pureza processado via ECAP, também encontraram elevada fração de contornos de baixo ângulo após o $1^{\circ}$ passe, perto de $89 \%$.

É possível também observar nas imagens referentes ao $1^{\circ}$ passe que ocorreu um severo refinamento da microestrutura, juntamente com o surgimento de subgrãos, podendo-se sugerir também uma recristalização dinâmica. Medidas de tamanho médio de grão realizado por EBSD confirmam tal observação. A secção transversal apresentou tamanho médio de grão 3,29 $\pm 4,53 \mu \mathrm{m}$, quando se compara esse valor ao encontrado no material de partida, observa-se uma redução no tamanho de grãos de aproximadamente $80 \%$, valor realmente muito elevado. Todavia nota-se observando o desvio padrão da medida de tamanho de grão e a Figura 3 que o material ainda apresenta elevada heterogeneidade, tal fator é proveniente do material de partida.

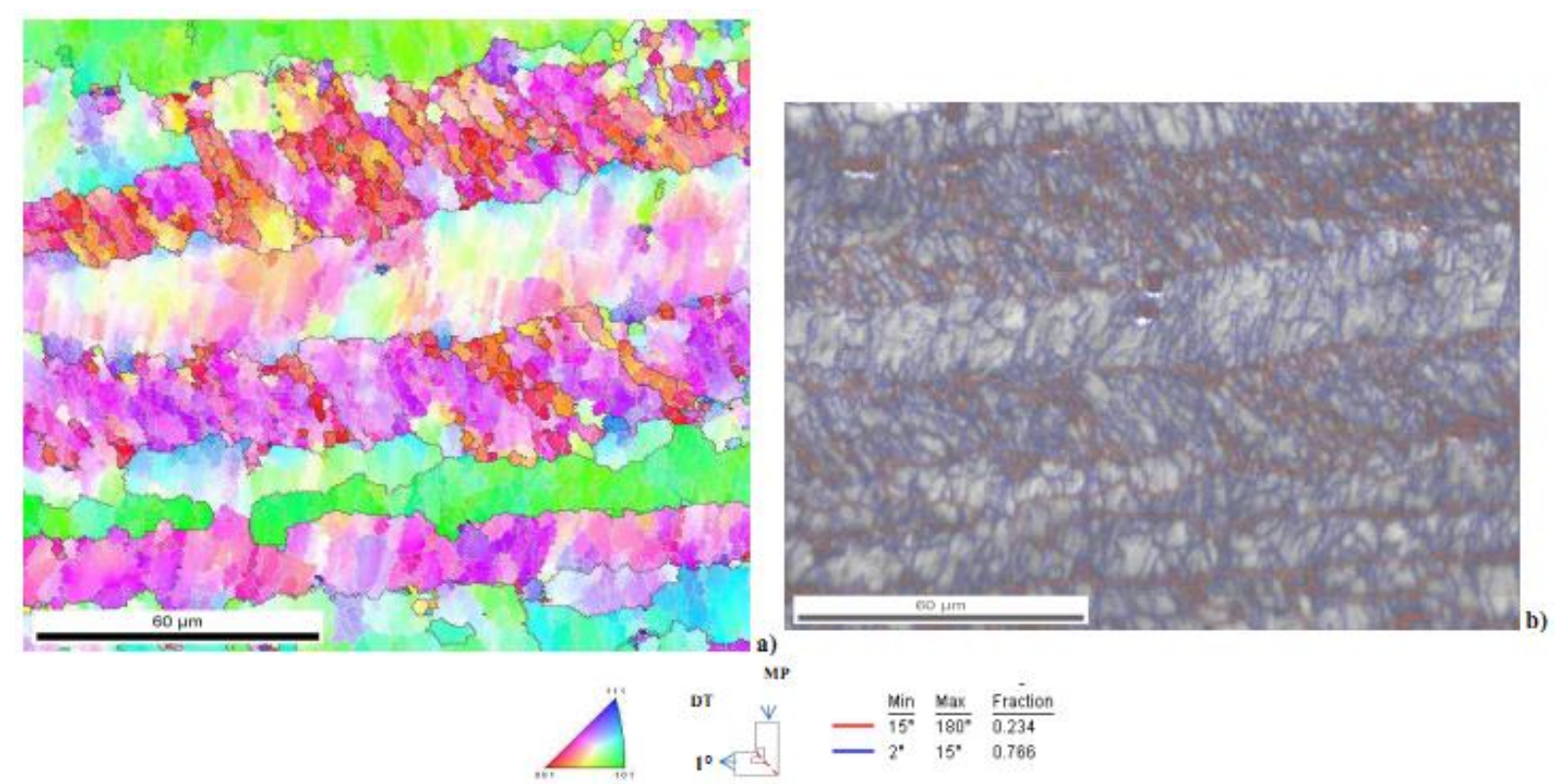

Figura 3. Mapa de orientação após $01^{\circ}$ passe, seção transversal; b) Mapa de qualidade com contornos delineados. (MEV - EBSD, 20 kV). Mapa de Dureza - Material de partida (Secção transversal). [9]

A dureza média encontrada foi de 54,1 $\pm 3,0$ Vickers bem acima de 31,1 $\pm 1,9$ Vickers encontrado para o material de partida, o que sugere um aumento extremamente elevado na energia interna do material. Este elevado aumento na energia interna do material é possivelmente decorrente do refinamento microestrutural e do surgimento de uma elevada quantidade de contornos de baixo ângulo. Esse resultado é coerente com os dados apresentados por Kawasaki e seus colaboradores [8].

\footnotetext{
* Contribuição técnica ao $69^{\circ}$ Congresso Anual da ABM - Internacional e ao 14ํㅡㄹ ENEMET - Encontro Nacional de Estudantes de Engenharia Metalúrgica, de Materiais e de Minas, 21 a 25 de julho de 2014, São Paulo, SP, Brasil.
} 


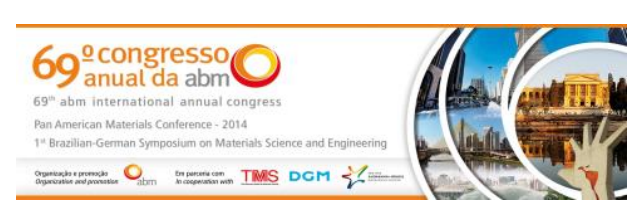

\subsection{Segundo Passe de Deformação - Rota C}

Dados quantitativos referentes ao $2^{\circ}$ passe foram obtidos da secção transversal, após processamento completo. Conforme apresentado na Figura 4 observa-se uma redução relevante de contornos de baixo ângulo quando comparado ao $1^{\circ}$ passe, $46 \%$ baixo ângulo e $54 \%$ alto ângulo.

Conforme apresentado por Resende [6], o refinamento de grãos após o $2^{\circ}$ passe ainda é intenso. Observa-se um tamanho médio de grão de 1,6 $\pm 1,7 \mu \mathrm{m}$. Nas áreas analisadas houve uma maior incidência de formação de subestruturas, sugerindo uma possível recristalização dinâmica. Este resultado pode estar relacionado ao fato de o material possuir baixas concentrações de solutos e elevado nível de energia armazenada, em virtude da deformação severa anterior, estes fatores contribuem para disparar o processo de recristalização. Comparando-se este valor ao encontrado no $1^{\circ}$ passe, conclui-se que a redução do tamanho de grão foi de aproximadamente $54 \%$, esta redução já se era esperada e se encontra em conformidade com estudos realizados por Kawasaki e seus colaboradores [8] e Rezende e colaboradores [6].

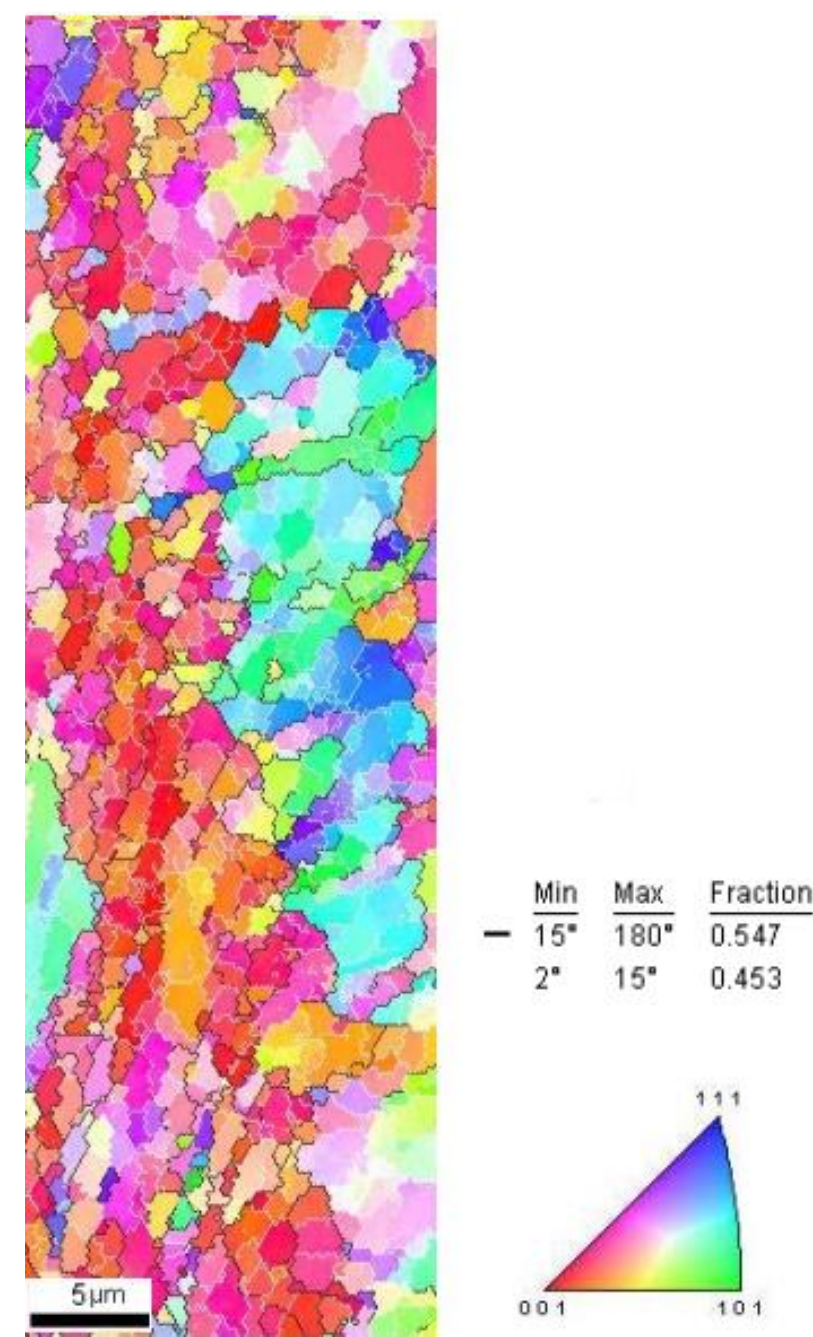

Figura 4. Mapa de orientação após o $2^{\circ}$ passe, seção transversal; (MEV - EBSD, 20 kV).

Após o segundo passe via ECAP observa-se uma redução nos valores médios de dureza Vickers, apresentando um valor médio de 47,5 \pm 1,8 Vickers, mostrando que

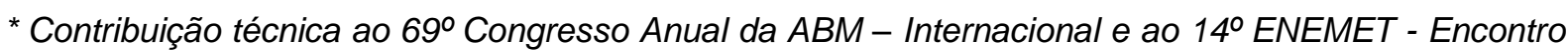
Nacional de Estudantes de Engenharia Metalúrgica, de Materiais e de Minas, 21 a 25 de julho de 2014, São Paulo, SP, Brasil. 


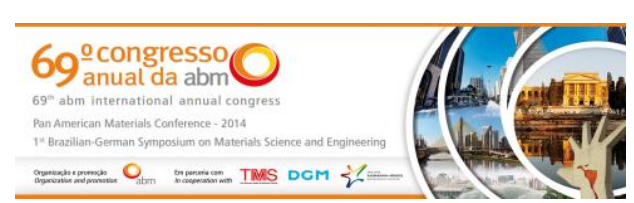

o passe que possui maior dureza é o primeiro passe de deformação, onde há também maior incidência de contornos de baixo ângulo. A Figura 5 apresenta uma comparação dos mapas de dureza das secções transversal do material de partida, do primeiro e do segundo passe de deformação, onde é possivel observar que não há mudança na hetereogeneidade da distribuição de valores de dureza, mostrando que após sucessos passes a microestrutura não tende a se homogeinizar. Isto pode ser explicado pelo aumento do número de contornos de alto ângulo causado pelo refinamento microestrutural, destacado por Gazdera et al em [9].

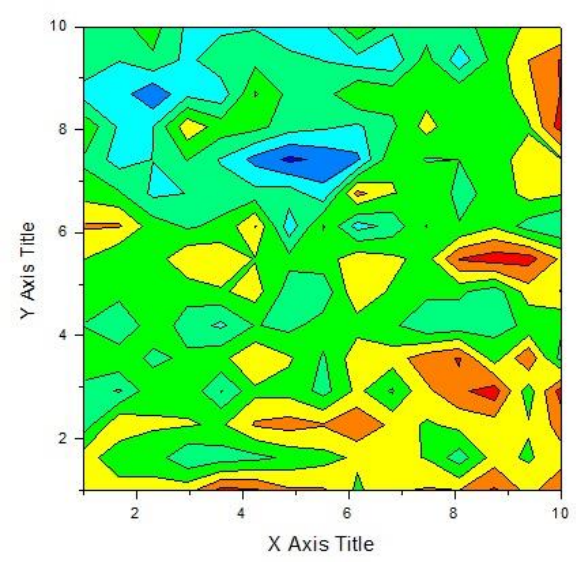

(a)
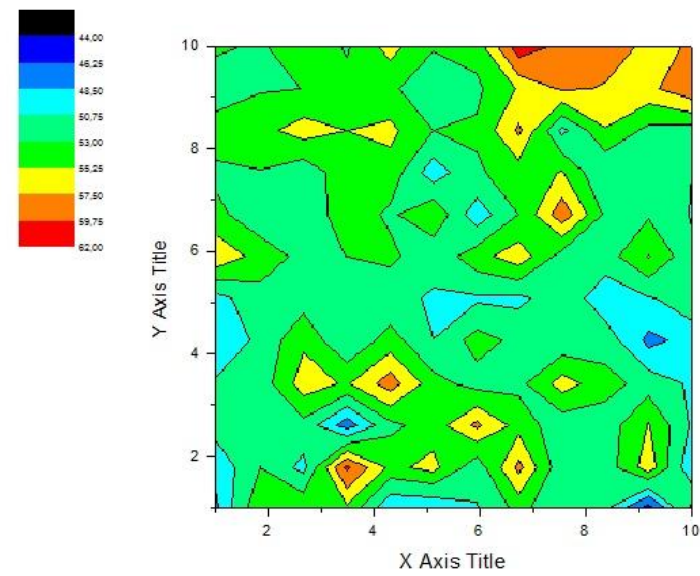

(b)
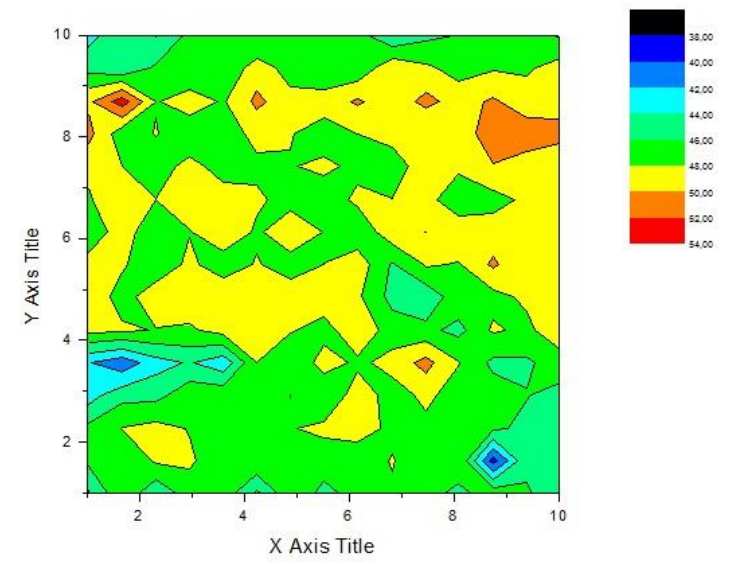

(c)

Figura 5. Mapas de Dureza - Material de partida (a); Primeiro passe (b); Segundo passe (c).

\section{CONCLUSÃo}

O comportamento do material a cada etapa de processamento de deformação plástica foi analisado sistematicamente. Pode-se concluir o refinamento microestrutural após o processamento via ECAP foi realizado eficientemente. Os resultados obtidos sugerem que os mecanismos responsáveis pela redução do tamanho de grão foram decorrentes de processos de subdivisão por meio de fragmentação e rotação de grãos. O processo se mostrou eficiente quanto ao refinamento dos grãos no decorrer dos passes.

Em relação ao comportamento mecânico, foi observado um crescimento na energia interna do material, constatado pelo seu aumento de dureza, cerca de $54 \%$ aproximadamente no primeiro passe de deformação.

* Contribuição técnica ao $69^{\circ}$ Congresso Anual da ABM - Internacional e ao 14ํㅡㄹ ENEMET - Encontro Nacional de Estudantes de Engenharia Metalúrgica, de Materiais e de Minas, 21 a 25 de julho de 2014, São Paulo, SP, Brasil. 


\section{Agradecimentos}

À CAPES, CNPq e FAPERJ pelo apoio financeiro.

\section{REFERÊNCIAS}

1 Azushima A, Koop R, Korhonen A. Severe plastic deformations (SPD) processes for metals. CIRP Annals - Manufacturing Technology. 2008;57:716735.

2 Lins JFC. Caracterização Microestrutural da Liga AIAA1070 Deformada. Universidade Federal Fluminense; 2009.

3 Valiev RZ, Islamgaliev RK, Alexandrov IV. Bulk nanostructured materials from severe plastic deformation. Progress in Materials Science. 2000;45:103-189.

4 Segal VM, Reznikov VI, Drobyshevskiy AE, Kopylov VI. Russian Metall. 1981; p.1-99.

5 Tolaminejad B, Dehghani K. Microstructural characterization and mechanical properties of nanostructured AA1070 aluminum after equal channel angular extrusion. Materials \& Design. 2012;34:285-292.

6 Rezende MO. Caracterização Microestrutural e da Textura cristalográfica de uma liga de Alumínio AA1070 Deformada a Frio via Prensagem em canais Equiangulares [dissertação de mestrado]. Volta Redonda: UFF; 2011.

7 Alvi $\mathrm{MH}$, Cheong SW, Sune JP, Weiland H, Rollet AD. Cube texture in hot rolled aluminum alloy (AA1050) - Nucleation and Growth Behavior. Acta Materialia. 2008;56:3098-3108.

8 Kawasaki M, Horita Z, Langdon TG. Microstructural evolution in high purity aluminum processed by ECAP. Materials Science and Engineering $A$. 2009;524:143-150.

9 Gazdera AA, Cao W, Davies CHJ, Pereloma EV. An EBSD Investigation of Interstitial-Free Steel Subjected to Equal Channel Angular Extrusion. Materials Science and Engineering A. 2008;497:341-352.

* Contribuição técnica ao 69 Congresso Anual da ABM - Internacional e ao 14ํㅡㄹ ENEMET - Encontro Nacional de Estudantes de Engenharia Metalúrgica, de Materiais e de Minas, 21 a 25 de julho de 2014, São Paulo, SP, Brasil. 